\title{
COEFFICIENT BOUNDS FOR CONVEX FUNCTIONS OF BOUNDED TYPE
}

\author{
K.-J. WIRTHS \\ (Communicated by Irwin Kra) \\ In memory of Professor Doctor Ernst Peschl (1906-1986)
}

\begin{abstract}
A normalized univalent function is called convex of bounded type if the curvature of the curve bounding the image domain of the unit disc lies between two fixed positive numbers. Sharp bounds for the modulus of the second and the third Taylor coefficient of such functions are derived. The proof concerning the second coefficient is based on a maximum-minimum principle for locally univalent functions.
\end{abstract}

1. Introduction. Let $f$ be regular and locally univalent in $D=\{z|| z \mid<1\}$ and

$$
\kappa_{f}(z)=\frac{1}{\left|z f^{\prime}(z)\right|} \operatorname{Re}\left(1+\frac{z f^{\prime \prime}(z)}{f^{\prime}(z)}\right), \quad 0<|z|=r<1,
$$

denote the curvature of $f(\{z|| z \mid=r\})$ at the point $f(z)$. In [1] Goodman introduced the class $C V\left(R_{1}, R_{2}\right)$ of functions $f$ regular and convex in $D$, normalized by $f(0)=f^{\prime}(0)-1=0$ which fulfill

$$
0<R_{1} \leq \liminf _{|z| \rightarrow 1} 1 / \kappa_{f}(z) \leq \limsup _{|z| \rightarrow 1} 1 / \kappa_{f}(z) \leq R_{2}<\infty .
$$

He called such functions convex of bounded type and discussed the coefficient problem for $C V\left(R_{1}, R_{2}\right)$ in [1 and 2]. Among other things he proved that for $f(z)=z+\sum_{k=2}^{\infty} a_{k} z^{k} \in C V\left(R_{1}, R_{2}\right)$ the inequalities

$$
\left|a_{k}\right| \leq\left(1-1 / R_{2}\right)^{(k-1) / 2}
$$

cannot be valid for all $k \geq 2$ and all $R_{2}>1$.

The aim of this paper is to give new proofs for (2) in the cases $k=2$ and $k=3$. In fact, (2) for $k=2$ was proved by Raupach in [6] as part of a longer discussion of the maximum principle of Peschl (see for instance [4 and 5]). In the sequel we shall give an independent proof of (2) based on a maximum-minimum principle for locally univalent functions.

In the case $k=3(2)$ is a simple consequence of an inequality proved in [7], where it was part of a longer investigation, too. Here we shall present a short proof of that inequality. Since in these proofs the use of the automorphisms of $D$ is essential we prefer to deal with nonnormalized functions.

Received by the editors October 9, 1986 and, in revised form, April 10, 1987.

1980 Mathematics Subject Classification (1985 Revision). Primary 30C50; Secondary 30C45.

Key words and phrases. Convex functions, bounded type, coefficient bounds. 
DEFinition. Let $K>0$ and $f$ be regular and locally univalent in $D$. Then $f$ is said to belong to the class $C(K)$ if and only if

$$
\liminf _{|z| \rightarrow 1} \kappa_{f}(z) \geq K
$$

REMARK. As the referee remarked one may choose another equivalent definition of the class $C(K)$. This one is based on a theorem due to Peschl on the curvature of images of circles under locally univalent mappings (see [3, Hauptsatz 1]). For our purposes we need only the following special case of that theorem:

Let $f \in C(K), K>0$. Then $\kappa_{f}$ has no local minimum in $D \backslash\{0\}$ and $\kappa_{f}(z)>K$ for $z \in D \backslash\{0\}$.

For convenience we give an outline of the proof (the idea of this proof was mentioned by Peschl in $[3, \S 3])$ : Let $\kappa_{f}\left(z_{1}\right), 0<\left|z_{1}\right|<1$, be a local minimum of $\kappa_{f}$. Then

$$
0=\frac{\partial}{\partial z} \kappa_{f}\left(z_{1}\right)=\frac{1}{2\left|z f^{\prime}(z)\right|}\left(\frac{z f^{\prime \prime}(z)}{f^{\prime}(z)}\right)^{\prime}-\left.\frac{1}{2 z}\left(1+\frac{z f^{\prime \prime}(z)}{f^{\prime}(z)}\right) \kappa_{f}(z)\right|_{z=z_{1}}
$$

Hence

$$
\frac{\partial^{2}}{\partial \bar{z} \partial z} \kappa_{f}\left(z_{1}\right)=-\left.\left|\frac{1}{2 z}\left(1+\frac{z f^{\prime \prime}(z)}{f^{\prime}(z)}\right)\right|^{2} \kappa_{f}(z)\right|_{z=z_{1}} .
$$

$\kappa_{f}(z)>0$ for $z \in D \backslash\{0\}$ because $f$ is a convex function. Therefore the last equality implies $\partial^{2} \kappa_{f}\left(z_{1}\right) / \partial \bar{z} \partial z<0$ which is a contradiction to our assumption. So the first assertion is proved. Since $\lim _{z \rightarrow 0} \kappa_{f}(z)=\infty \kappa_{f}$ cannot be constant. This together with the first part proves $\kappa_{f}(z)>K$ for $z \in D \backslash\{0\}$. Therefore we get for $f$ regular and locally univalent in $D: f \in C(K), K>0$, if and only if $\kappa_{f}(z)>K$ for $z \in$ $D \backslash\{0\}$.

From these observations the following implication which we shall need in the sequel is easily derived: If $f \in C(K), K>0$, and $f_{r}(z)=f(r z), 0<r<1$, then $f_{r} \in C\left(K^{\prime}\right) \subset C(K)$ for a $K^{\prime}>K$.

Obviously $C V\left(R_{1}, R_{2}\right) \subset C\left(1 / R_{2}\right)$. It may be worthwhile to notice that it is impossible to replace the boundary condition concerning $R_{1}$ in the definition of $C V\left(R_{1}, R_{2}\right)$ by a global condition in a similar manner as it is done above for $C\left(1 / R_{2}\right)$. This again is a consequence of $\lim _{z \rightarrow 0} \kappa_{f}(z)=\infty$.

\section{The sharp bound for $\left|a_{2}\right|$.}

THEOREM 1. Let $f$ be regular at $z_{0} \in D$. (a) If $f^{\prime}\left(z_{0}\right) \neq 0$, the function

$$
u_{f}(z)=\frac{1-\left|\left(f^{\prime \prime}(z) / 2 f^{\prime}(z)\right)\left(1-|z|^{2}\right)-\bar{z}\right|^{2}}{\left|f^{\prime}(z)\right|\left(1-|z|^{2}\right)}
$$

defined in a neighborhood of $z_{0}$ has a local minimum at $z_{0}$ if and only if $f$ is a Möbius transformation.

(b) If $f^{\prime}\left(z_{0}\right) \neq 0$ and $f^{\prime \prime}\left(z_{0}\right)\left(1-\left|z_{0}\right|^{2}\right) \neq 2 \bar{z}_{0} f^{\prime}\left(z_{0}\right), u_{f}$ has a local maximum at $z_{0}$ if and only if $f$ is a Möbius transformation.

PROOF. (a) Let

$$
\tilde{f}(z)=f\left(\frac{z+z_{0}}{1+\bar{z}_{0} z}\right)
$$


Since $\tilde{f}^{\prime}(0)=f^{\prime}\left(z_{0}\right)\left(1-\left|z_{0}\right|^{2}\right)$ and

$$
u_{f}\left(\frac{z+z_{0}}{1+\bar{z}_{0} z}\right)=u_{\tilde{f}}(z)
$$

we only have to prove the assertion for $z_{0}=0$. Let $f(z)=\sum_{k=0}^{\infty} a_{k} z^{k}, a_{1} \neq 0$. Here as always in this proof $z$ has to be chosen from a suitable neighborhood of the origin. The assumption that $u_{f}$ has a local minimum at the origin leads to the inequality

(4) $0 \leq h_{f}(z):=1-\left|\frac{f^{\prime \prime}(z)}{2 f^{\prime}(z)}\left(1-|z|^{2}\right)-\bar{z}\right|^{2}-\left(1-\left|\frac{a_{2}}{a_{1}}\right|^{2}\right)\left|\frac{f^{\prime}(z)}{a_{1}}\right|\left(1-|z|^{2}\right)$.

Now we prove by mathematical induction on $k, k \geq 2$, that (4) implies

$$
a_{k}=\left(a_{2} / a_{1}\right)^{k-1} a_{1}, \quad k \geq 2 .
$$

Since any Möbius transformation $g$ regular at the origin may be written as

$$
g(z)=a_{0}+\frac{a_{1} z}{1-\left(a_{2} / a_{1}\right) z}
$$

this is just the assertion.

For $k=2(5)$ is trivial. From now on we use the abbreviation $A_{k}=a_{k} / a_{1}$ and assume $A_{l}=\left(A_{2}\right)^{l-1}$ for $2 \leq l \leq k$. We first deal with the case $A_{2} \neq 0$ and we get

$$
\begin{aligned}
\frac{f^{\prime}(z)}{a_{1}} & =\frac{1}{\left(1-A_{2} z\right)^{2}}+o\left(|z|^{k-1}\right), \\
\frac{f^{\prime \prime}(z)}{2 f^{\prime}(z)} & =\frac{A_{2}}{1-A_{2} z}+\frac{1}{2} k(k+1)\left(A_{k+1}-\left(A_{2}\right)^{k}\right) z^{k-1}+o\left(|z|^{k-1}\right) .
\end{aligned}
$$

A comparison between the expansions of $h_{f}$ and $h_{g}$ around the origin where $g$ is as in (6) yields

$$
h_{f}(z)-h_{g}(z)=-\operatorname{Re} k(k+1)\left(A_{k+1}-\left(A_{2}\right)^{k}\right) \bar{A}_{2} z^{k-1}+o\left(|z|^{k-1}\right) .
$$

Because of the identity $h_{g}(z) \equiv 0$ this together with (4) leads to

$$
\operatorname{Re}\left(A_{k+1}-\left(A_{2}\right)^{k}\right) \bar{A}_{2} z^{k-1} \leq 0
$$

and therefore to the desired conclusion $A_{k+1}=\left(A_{2}\right)^{k}$. Now let $A_{2}=0$. From

$$
h_{f}(z)=3 \operatorname{Re} A_{3} z^{2}-9\left|A_{3}\right|^{2}|z|^{2}+o\left(|z|^{2}\right) \geq 0
$$

we conclude $A_{3}=0$. The assumption $A_{l}=0$ for $2 \leq l \leq k, k \geq 3$, and (4) imply

$$
h_{f}(z)=(k-1)(k+1) \operatorname{Re} A_{k+1} z^{k}+o\left(|z|^{k}\right) \geq 0
$$

and therefore $A_{k+1}=0$. This completes the proof of (a). The proof of (b) is analogous to that part of the above proof wherein $A_{2} \neq 0$.

As an application of Theorem 1 we get

THEOREM 2 (CF. [1, 2 AND 6]). (a) For $f \in C(K), K>0$, the inequality

$$
\left|\frac{f^{\prime \prime}(z)}{2 f^{\prime}(z)}\left(1-|z|^{2}\right)-\bar{z}\right|^{2} \leq 1-K\left|f^{\prime}(z)\right|\left(1-|z|^{2}\right)
$$


is valid for all $z \in D$. Equality for one $z \in D$ implies equality for all $z \in D$ and this can happen if and only if

$$
f(z)=\frac{e^{i \phi}}{K}\left(\frac{z+a}{1+\bar{a} z}\right)+b, \quad \phi \in \mathbf{R},|a|<1, b \in \mathbf{C} .
$$

(b) If $f(z)=\sum_{k=0}^{\infty} a_{k} z^{k} \in C(K), K>0$, then

$$
\left|a_{2} / a_{1}\right|^{2} \leq 1-K\left|a_{1}\right| \text {. }
$$

Equality occurs only for the functions (8).

(c) If $f(z)=z+\sum_{k=2}^{\infty} a_{k} z^{k} \in C V\left(R_{1}, R_{2}\right),\left|a_{2}\right|^{2} \leq 1-1 / R_{2}$. Equality occurs only for the functions

$$
f(z)=z /(1+a z), \quad R_{2}\left(1-|a|^{2}\right)=1 .
$$

PROOF. (a) According to the above remark we may assume $f^{\prime}(z) \neq 0$ for $z \in \bar{D}$. For such functions $f \in C(K)$ an immediate consequence of Theorem 1 is

$$
\begin{aligned}
u_{f}(z) & \geq \liminf _{|z| \rightarrow 1} u_{f}(z) \\
& =\liminf _{|z| \rightarrow 1} \frac{1}{\left|f^{\prime}(z)\right|}\left(\operatorname{Re}\left(1+\frac{z f^{\prime \prime}(z)}{f^{\prime}(z)}\right)+\frac{1}{4}\left|\frac{f^{\prime \prime}(z)}{f^{\prime}(z)}\right|^{2}\left(1-|z|^{2}\right)\right) \\
& =\liminf _{|z| \rightarrow 1} \kappa_{f}(z) \geq K \quad \text { for } z \in D .
\end{aligned}
$$

From this we conclude that (7) is valid for all $f \in C(K)$ and all $z \in D$. Hence $u_{f}(z) \geq K$ for all $f \in C(K)$ and all $z \in D$. If $u_{f}\left(z_{1}\right)=K$ for one $z_{1} \in D u_{f}$ has a local minimum at $z_{1}$. In this case $f$ is a Möbius transformation by Theorem 1 and $u_{f}$ is constant. The only Möbius transformations for which $u_{f}(z) \equiv K$ are the functions (8). (9) is (7) for $z=0$ and (c) follows immediately from (b).

III. The sharp bound for $\left|a_{3}\right|$.

THEOREM 3 (COMPARE [1, 2 AND 7]). (a) Let $f \in C(K), K>0$ and

$$
[f]_{z}(z)=\frac{f^{\prime \prime \prime}(z)}{f^{\prime}(z)}-\frac{3}{2}\left(\frac{f^{\prime \prime}(z)}{f^{\prime}(z)}\right)^{2}
$$

denote the Schwarzian derivative of $f$. Then the inequality

$$
\begin{aligned}
& \frac{1}{2}\left|[f]_{z}(z)\right|\left(1-|z|^{2}\right)^{2}\left(1-\frac{K}{2}\left|f^{\prime}(z)\right|\left(1-|z|^{2}\right)\right) \\
& \quad \leq 1-\left|\frac{f^{\prime \prime}(z)}{2 f^{\prime}(z)}\left(1-|z|^{2}\right)-\bar{z}\right|^{2}-K\left|f^{\prime}(z)\right|\left(1-|z|^{2}\right)
\end{aligned}
$$

is valid for all $z \in D$. Equality for one $z \in D$ implies equality for all $z \in D$ and this can happen if and only if $f$ is of the form (8).

(b) Let $f(z)=\sum_{k=0}^{\infty} a_{k} z^{k} \in C(K), K>0$. Then

$$
3\left|\frac{a_{3}}{a_{1}}-\left(\frac{a_{2}}{a_{1}}\right)^{2}\right| \leq \frac{1-\left|a_{2} / a_{1}\right|^{2}-K\left|a_{1}\right|}{1-K\left|a_{1}\right| / 2}
$$

and

$$
\left|a_{3} / a_{1}\right| \leq 1-K\left|a_{1}\right| .
$$

Equality occurs in both cases if and only if $f$ is of the form (8). 
(c) Let $f(z)=z+\sum_{k=2}^{\infty} a_{k} z^{k} \in C V\left(R_{1}, R_{2}\right)$. Then

and

$$
3\left|a_{3}-\left(a_{2}\right)^{2}\right| \leq \frac{1-\left|a_{2}\right|^{2}-1 / R_{2}}{1-1 / 2 R_{2}}
$$

$$
\left|a_{3}\right| \leq 1-1 / R_{2} .
$$

Equality occurs in both cases if and only if $f$ is of the form (10).

Proof. (a) First we consider functions $f \in C(K)$ that fulfill $\left|f^{\prime}(z)\right|>0$ and $u_{f}(z)-K>0$ for $z \in \bar{D}$. According to the above remark and Theorem 2 these inequalities are correct for $f_{r}$ if $f \in C(K)$ and $0<r<1$. Under these circumstances

$$
v_{f}(z)=\frac{\frac{1}{2}\left|[f]_{z}(z)\right|\left(1-|z|^{2}\right)^{2}\left(1-(K / 2)\left|f^{\prime}(z)\right|\left(1-|z|^{2}\right)\right)}{\left(u_{f}(z)-K\right)\left|f^{\prime}(z)\right|\left(1-|z|^{2}\right)}
$$

is continuous on $\bar{D}$ and $\lim _{|z| \rightarrow 1} v_{f}(z)=0$.

We show that the assumption $v_{f}\left(z_{0}\right) \geq 1$ at a point $z_{0}$ where $v_{f}$ has a local maximum leads to a contradiction. Considerations analogous to those used in the proof of Theorem 1 show that it is no restriction to take $z_{0}=0$. Let $a_{k}, A_{k}$ be defined as above. We may formulate our assumption as follows:

$$
\begin{aligned}
0 \leq & H_{f}(z):=\left(1-\frac{K}{2}\left|a_{1}\right|\right)\left(u_{f}(z)-K\right)\left|f^{\prime}(z)\right|\left(1-|z|^{2}\right) \\
& -\left(1-\left|A_{2}\right|^{2}-K\left|a_{1}\right|\right) \frac{1}{2}\left|[f]_{z}(z)\right|\left(1-|z|^{2}\right)^{2} \frac{\left(1-(K / 2)\left|f^{\prime}(z)\right|\left(1-|z|^{2}\right)\right)}{3\left|A_{3}-\left(A_{2}\right)^{2}\right|}
\end{aligned}
$$

for $z$ in a neighborhood of the origin and

$$
v_{f}(0)=\frac{3\left|A_{3}-\left(A_{2}\right)^{2}\right|\left(1-(K / 2)\left|a_{1}\right|\right)}{1-\left|A_{2}\right|^{2}-K\left|a_{1}\right|} \geq 1 .
$$

Expansion of $H_{f}$ around the origin leads to

$$
H_{f}(z)=\operatorname{Re} P z+Q|z|^{2}+\operatorname{Re} R z^{2}+o\left(|z|^{2}\right) \geq 0,
$$

where

$$
\begin{aligned}
P= & \left(2-K\left|a_{1}\right|\right)\left(A_{2}+2\left|A_{2}\right|^{2} A_{2}-3 A_{3} \bar{A}_{2}\right)-\left(1+\left|A_{2}\right|^{2}\right) K\left|a_{1}\right| A_{2} \\
& -\left(1-\left|A_{2}\right|^{2}-K\left|a_{1}\right|\right)\left(1-\frac{K}{2}\left|a_{1}\right|\right) S \\
S= & \left(4 A_{4}-8 A_{3} A_{2}+4\left(A_{2}\right)^{3}\right) /\left(A_{3}-\left(A_{2}\right)^{2}\right), \\
Q= & \left(1-\frac{K}{2}\left|a_{1}\right|\right)\left(2\left|A_{2}\right|^{2}-1-\left|3 A_{3}-2\left(A_{2}\right)^{2}\right|^{2}-K\left|a_{1}\right|\left(\left|A_{2}\right|^{2}-1\right)\right) \\
& -\left(1-\left|A_{2}\right|^{2}-K\left|a_{1}\right|\right)\left(\frac{1}{4}\left(1-\frac{K}{2}\left|a_{1}\right|\right)|S|^{2}-\frac{K}{2}\left|a_{1}\right| \operatorname{Re} S \bar{A}_{2}\right. \\
& \left.\quad-\left(2-K\left|a_{1}\right|\right)-\frac{K}{2}\left|a_{1}\right|\left(\left|A_{2}\right|^{2}-1\right)\right) .
\end{aligned}
$$

The explicit form of $R$ is of no interest. (14) implies $P=0$ and $Q \geq 0$. Now we solve $P=0$ for $S$ and insert the obtained expression into (15). After a straightforward calculation we see that $Q \geq 0$ is equivalent to

$$
\frac{1-\left|A_{2}\right|^{2}-K\left|a_{1}\right|}{1-(K / 2)\left|a_{1}\right|}\left(1-\left(v_{f}(0)^{2}\right)\left(1-K\left|a_{1}\right|\right)-\frac{K}{2}\left|a_{1}\right|\left(1-\left|A_{2}\right|^{2}-K\left|a_{1}\right|\right)\right) \geq 0 .
$$


The assumption $v_{f}(0) \geq 1$ obviously implies

$$
-(K / 2)\left|a_{1}\right|\left(1-\left|A_{2}\right|^{2}-K\left|a_{1}\right|\right)^{2} \geq 0
$$

which is a contradiction for the functions under consideration. This proves that (11) is correct under the special conditions mentioned in the beginning of the proof and that under these circumstances equality cannot occur. Since $\lim _{r \rightarrow 1} f_{r}(z)=f(z)$ this proves (11) for any $f \in C(K)$. Now we shall discuss equality in (11): If there is a $z \in D$ for which the right-hand side of (11) equals zero, $f$ is a Möbius transformation and both sides of (11) are vanishing on $D$. If $u_{f}(z)-K>0$ for all $z \in D, v_{f}$ is defined on $D$ and $v_{f}(z) \leq 1$ for $z \in D$ as we have seen before. Hence $\lim \sup _{|z| \rightarrow 1} v_{f}(z) \leq 1$ and equality in (11) at a point $z_{1} \in D$ therefore would mean that $v_{f}$ would attain a local maximum at $z_{1}$ and $v_{f}\left(z_{1}\right)=1$. This leads to a contradiction in the same way as before. (11) for $z=0$ is (12). (13) follows from (12) by use of the triangle inequality and (9). (b) immediately yields (c).

\section{REFERENCES}

1. A. W. Goodman, Convex functions of bounded type, Proc. Amer. Math. Soc. 92 (1984), 541546.

2. - More on convex functions of bounded type, Proc. Amer. Math. Soc. 97 (1986), 303-306.

3. E. Peschl, Über die Krümmung von Niveaukurven bei der konformen Abbildung einfachżusammenhängender Gebiete auf das Innere eines Kreises, Math. Ann. 106 (1932), 574-594.

4. L Les invariants différentiels non holomorphes et leur rôle dans la théorie des fonctions, Rend. Sem. Mat. Messina 1 (1955), 100-108.

5. $\_$Über die Verwendung von Differentialinvarianten bei gewissen Funktionenfamilien und die Übertragung einer darauf gegründeten Methode auf partielle Differentialgleichungen, Ann. Acad. Sci. Fenn. Ser. A 1 (1963), 336/6.

6. E. Raupach, Eine Abschätzungsmethode für die reellwertigen Lösungen der Differentialgleichung $\Delta \alpha=-4 /(1-z \bar{z})^{2}$, Bonner Math. Schriften, No. 9 (1960).

7. K.-J. Wirths, Verallgemeinerungen eines Maximumprinzips, Bonner Math. Schriften, No. 51 (1971).

INSTITUT FÜR ANALYSIS, TECHNISCHE UNIVERSITÄT BRAUNSCHWEIG, D-3300 BRAUNSCHWEIG, FEDERAL REPUBLIC OF GERMANY 